\title{
Transfert de gènes dans les hépatocytes à l'aide de vecteurs rétroviraux
}

Le foie constitue une cible privilégiée pour un transfert de gènes à visée thérapeutique. Il est le siège de nombreux dérèglements héréditaires graves qui ne pourraient être corrigés que dans le contexte de la fonction hépatique (et donc dans les hépatocytes eux-mêmes). C'est le cas, par exemple, de certaines formes d'hypercholestérolémie familiale secondaires à des mutations touchant le gène du récepteur des LDL. Dans le cas de déficits touchant une fonction extrahépatique, le transfert dans le foie d'un gène devant exercer son effet à distance pourrait également se révéler efficace. Le rôle physiologique du foie, pourvoyeur de la plupart des protéines plasmatiques et organe de détoxification, le place en effet en position intéressante comme siège de l'expression d'un gène étranger introduit dans un but thérapeutique.

Une approche envisageable consisterait à effectuer le transfert de gène sur des hépatocytes prélevés après traitement du foie par la collagénase et mis en culture. Pour juger de la faisabilité de cette technique, nous avons défini des conditions de culture permettant une infection des hépatocytes de rat par des vecteurs rétroviraux amphotropes véhiculant le gène de la ß-galactosidase d'E. coli Nous utilisons le gène nlslac $Z$ [1], qui code pour une enzyme à localisation nucléaire. Ceci nous permet de réaliser des doubles colorations qui révèlent l'activité du gène transféré par une coloration nucléaire et l'existence de marqueurs hépatocytaires par une coloration cytoplasmique (peroxydase, glucose-6-phosphatase). Ainsi pouvons-nous affirmer que les cellules exprimant le gène transféré sont bien des hépatocytes, et non des cellules biliaires ou endothéliales qui contaminent fréquemment ces cultures. En effectuant l'infection trois jours après la mise en culture en parvenons à infecter $10 \%$ à $15 \%$ des hépatocytes. Nous avons ensuite cherché à réimplanter ces hépatocytes modifiés dans l'organisme. Alors que des cellules fraîchement explantées sont capables de former un tissu hépatique après réinjection [2], nos tentatives pour reproduire ce phénomène à partir de cellules maintenues quelques jours en culture ont toujours échoué.

Nous nous sommes alors orientés vers une autre approche dans laquelle nous avons cherché à obtenir l'infection d'hépatocytes in situ par des vecteurs rétroviraux. Ce travail a été mené en collaboration avec l'équipe chirurgicale du Pr D. Houssin (laboratoire de chirurgie expérimentale, CHU Cochin, Paris, France) [3]. Il est possible, chez le rat et la souris comme chez l'homme, d'exclure temporairement le foie de la circulation et de le perfuser sélectivement in situ [4]. Nous avons utilisé cette méthode chez le rat pour infecter des hépatocytes in vivo avec notre vecteur rétroviral portant nlslac $Z$. La perfusion est effectuée 24 heures après une hépatectomie de $70 \%$ qui provoque une régénération du tissu hépatique et facilite l'infection rétrovirale. Un volume d'environ $50 \mathrm{ml}$ de surnageant viral, contenant $10^{5}$ particules amphotropiques infectieuses par $\mathrm{ml}$ est perfusé avec un débit de 5 millilitre par minute. Les animaux sont sacrifiés 2 à 15 semaines après la perfusion, le foie est fixé in toto, et une réaction de détection de la B-galactosidase est effectuée sur des coupes à congélation.

Avec cette méthode, nous sommes capables de transférer le gène rapporteur dans les hépatocytes, et son expression peut être mise en évidence plusieurs mois après l'opération (le délai le plus long est actuellement de 9 mois). Les cellules intéressées par le transfert de gène présentent un noyau bleu après révé- lation de l'activité ß-galactosidase (figure 1). Elles apparaissent isolées ou en petites colonies de 2 à 10 cellules, réparties uniformément dans le parenchyme hépatique. Cet aspect suggère, en accord avec les travaux anciens sur la régénération hépatique, que tous les hépatocytes participent à ce processus [5-7]. De $1 \%$ à $5 \%$ des hépatocytes reçoivent et expriment le gène rapporteur, soit un total de 20 à 80 millions de cellules. Ce niveau d'efficacité permet d'envisager la distribution à l'organisme, à partir du foie, d'une protéine thérapeutique exprimant un transgène; voire l'élimination d'un métabolite toxique du sérum. Une des conditions requises pour l'utilisation thérapeutique éventuelle des techniques de transfert génétique est la possibilité de cibler un type cellulaire à l'exclusion de tous les autres. La procédure de perfusion du foie isolé du reste de l'organisme, associée à l'utilisation de vecteurs rétroviraux strictement défectifs (ou incompétents) pour la réplication, évite la propagation accidentelle du vecteur à un autre organe que le foie. Nous l'avons vérifié en utilisant un test de PCR capable de révéler une copie du transgène dans 10000 cellules. Alors que le transgène nlslac $Z$ était facilement mis en évidence par cette technique dans le tissu hépatique des animaux traités, il n'était pas détectable dans les autres tissus (poumons, rate, reins, cerveau).

Notre objectif est maintenant d'évaluer si le transfert de gène dans les hépatocytes, effectué dans ces conditions, s'accompagne d'effets secondaires indésirables à long terme, et s'il présente un intérêt thérapeutique. Pour cela, nous répétons ces expériences sur des séries importantes de rats qui ne seront analysés que dans plusieurs mois. Par ail- 


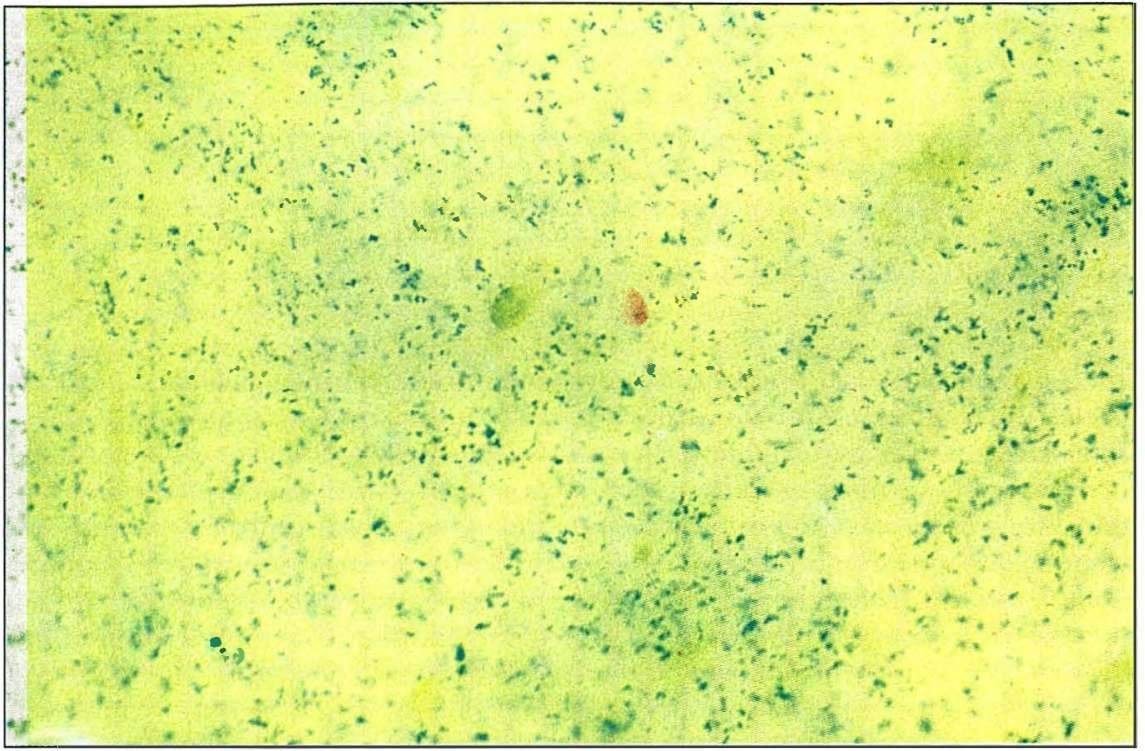

Figure 1. Coloration histochimique révélant l'activité $\beta$-galactosidase nucléaire dans les hépatocytes d'un animal traité un mois auparavant. Vingt-quatre heures après résection de $70 \%$ de la masse hépatique, le tissu hépatique restant a été perfusé avec une suspension contenant un vecteur rétroviral amphotrope qui véhicule le gène nlslac $\mathrm{Z}$.

leurs, nous cherchons à corriger le déficit métabolique du rat Gunn dont l'enzyme de conjugaison de la bilirubine (bilirubine UDPGT) est défectueuse. Un ADNc codant pour cette enzyme a été récemment isolé [8], et nous l'avons introduit dans des vecteurs rétroviraux. Les rats Gunn présentent un ictère sévère à bilirubine non conjuguée - rarement accompagné d'atteinte neurologique. Ce modèle animal est homologue à la très rare maladie de Crigler-Najjar, pour laquelle une transplantation hépatique est souvent indiquée.

La mise en œuvre de ce protocole de transfert de gène dans les hépatocytes est techniquement réalisable chez l'homme. L'approche chirurgicale a déjà été utilisée, et les hépatocytes humains en culture sont infectables par des rétrovirus (N. Ferry, résultats non publiés). Elle ne sera toutefois envisagée qu'après démonstration de son efficacité thérapeutique et de son innocuité chez le rat, ainsi que de sa faisabilité chez un gros mammifere.

N. F.

O. D. J.-M. H.
1. Kalderon D, Roberts BL, Richardson WD, Smith AE. A short amino acid sequence able to specify nuclear location. Cell 1984; 39 : 499-509.

2. Ponder KP, Gupta S, Leland F, et al. Mouse hepatocytes migrate to liver parenchyma and function indefinitely after intrasplenic transplantation. Proc Natl Acad Sci USA 1991; 88 : 1217-21.

3. Ferry N, Duplessis O, Houssin D, Danos O, Heard JM. Retroviral-mediated gene transfer into hepatocytes in vivo. Proc Natl Acad Sci USA 1991; 88 : 8377-81.

4. Adam R, Poggi L, Capron M, et al. A sanguineous isolated hyperthermic in vivo perfusion of the liver in the rat. Eur Surg Res 1987 ; 19 : 366-74.

5. Fabrikant JI. The kinctics of cellular proliferation in regenerating liver. J Cell Biol $1968 ; 36$ : 551-65.

6. Grishaw JW. A morphologic study of deoxyribonucleic acid synthesis and cell proliferation in regenerating rat liver; autoradiography with thymidine-H-3. Cancer Res $1962 ; 22: 842-9$

7. Rabes HM, Wirshing R, Tuczek HV, Iseler G. Analysis of cell cycle compartments of hepatocytes after partial hepatectomy. Cell

8. Sato $\mathrm{H}$ Koiwai $\mathrm{O}$, Tanabe $\mathrm{K}$, Kashiwamata $\mathrm{S}$. Isolation ans sequencing of rat liver bilirubin UDP-glucuronyltransferase cDNA : possible alternate splicing of a common primary transcript. Biochem Biophys Res Tissue Kinet 1976; 9 : 517-32 Commun $1990 ; 169: 260-4$.

\section{BRÈVE}

La CRH (corticotropinrcleasing hormone) est aussi un médiateur de l'inflammation. La CRH a été isolée de l'hypothalamus et identifiéc par ses propriétés de libérinc de l'ACTH, c'est-à-dirc de contrôleur central de l'axc hypothalomo-hypophyso-surrénalien. Cependant, les actions de la CRH ne se limitent pas à ce contrôle cettc hormone est également capable d'activer la production d'interlcukine 1 et 2 (IL-1 et IL-2) par des macrophages, de coopérer avec des lectines dans la stimulation de la prolifération lymphocytaire et d'augmenter l'expression des réceptcurs d'IL-2 à la surface des lymphocytes T. Des récepteurs de la CRH cxistent dans différentes souspopulations leucocytaires a niveau desquelles ont également été détectés de l'antigène et de l'ARN messager CRH. Tous ces résultats suggéraient que la $\mathrm{C} R \mathrm{H}$ pouvait intervenir sur la réponse inflammatoire. Plusieurs équipes américaines de Bethesda (MD, USA) [1] viennent maintenant de montrer que l'injection à un rat d'un anticorps antiCRH diminuait considérablement la réponse inflammatoire à unc injection de carrageénine (une substance inductrice d'une inflammation aseptique) : le volume de l'exsudat et l'afflux de cellules inflammatoires étaient réduits de $50 \%$ à $60 \%$, alors que le blocage de la $\mathrm{CRH}$ entraîne unc diminution de la réponse surrénalienne à l'inflammation, ce qui aurait pu laisser prévoir un effet inverse puisque les glucocorticoïdes sont des substances antiinflammatoires bien caractérisées. Un anticorps anti-TNF $\alpha$ (tumor necrosis factor) a approximativement les mêmes effets que l'anti-CRH, leurs actions n'étant pas additives. Une importante quantitć de CRH immunoréactive est détectée dans le tissu enflammé, sans augmentation parallèle de l'hormone circulante. La CRH semble donc être une cytokine jouant un rôle important dans la réponse inflammatoire au niveau des cellules effectrices périphériques.

[1. Caralis K, et al. Science 1991 254: 421-3.] 